\title{
Article \\ Numerical Simulation and Experimental Study of Capacitive Imaging Technique as a Nondestructive Testing Method ${ }^{\dagger}$
}

\author{
Farima Abdollahi-Mamoudan ${ }^{1, *(\mathbb{D})}$, Sebastien Savard ${ }^{2}$, Tobin Filleter ${ }^{3}\left(\mathbb{D}\right.$, Clemente Ibarra-Castanedo ${ }^{1}(\mathbb{D})$ and \\ Xavier P. V. Maldague ${ }^{1, *(D)}$
}

check for

updates

Citation: Abdollahi-Mamoudan, F.;

Savard, S.; Filleter, T.; Ibarra-

Castanedo, C.; Maldague, X.P.V.;

Numerical Simulation and

Experimental Study of Capacitive

Imaging Technique as a

Nondestructive Testing Method. Appl

Sci. 2021, 11, 3804. https://doi.org/

10.3390/app11093804

Academic Editor: Giosue Caliano

Received: 15 March 2021

Accepted: 16 April 2021

Published: 22 April 2021

Publisher's Note: MDPI stays neutra with regard to jurisdictional claims in published maps and institutional affiliations.

Copyright: (c) 2021 by the authors. Licensee MDPI, Basel, Switzerland. This article is an open access article distributed under the terms and conditions of the Creative Commons Attribution (CC BY) license (https:/ / creativecommons.org/licenses/by/ $4.0 /)$.
1 Department of Electrical and Computer Engineering, Université Laval, 1065 Avenue de la Médecine Québec, QC G1V 0A6, Canada; clemente.ibarra-castanedo@gel.ulaval.ca

2 Eddyfi Technologies, 3425, av. Pierre-Ardouin, Québec, QC G1P 0B3, Canada; ssavard@eddyfi.com

3 Department of Mechanical and Industrial Engineering, University of Toronto, 5 King's College, Toronto, ON M5S 3G8, Canada; filleter@mie.utoronto.ca

* Correspondence: farima.abdollahi-mamoudan.1@ulaval.ca (F.A.-M.); xavier.maldague@gel.ulaval.ca (X.P.V.M.)

$+\quad$ This paper is an extended version of the paper presented in Structural Health Monitoring and Non-destructive Testing 2020.

\begin{abstract}
It was recently demonstrated that a coplanar capacitive sensor could be applied to the evaluation of materials without the disadvantages associated with the other techniques. This technique effectively detects changes in the dielectric properties of the materials due to, for instance, imperfections or variations in the internal structure, by moving a set of simple electrodes on the surface of the specimen. An AC voltage is applied to one or more electrodes and signals are detected by others. This is a promising inspection method for imaging the interior structure of the numerous materials, without the necessity to be in contact with the surface of the sample. In this paper, finite element (FE) modeling was employed to simulate the electric field distribution from a coplanar capacitive sensor and the way it interacts with a nonconducting sample. Physical experiments with a prototype capacitive sensor were also performed on a Plexiglas sample with subsurface defects, to assess the imaging performance of the sensor. A good qualitative agreement was observed between the numerical simulation and experimental result.
\end{abstract}

Keywords: coplanar sensor; capacitive sensing; NDT techniques; FE modeling; defects

\section{Introduction}

There are several methods for evaluating the integrity of materials, and an important category of them is nondestructive evaluation (NDE) or nondestructive testing (NDT) methods. This field includes identifying and characterization the flaw on the surface and under the surface of materials without cutting apart or altering the material [1]. It means NDT refers to the process of evaluating and inspecting materials to identify or detect defects in comparison with some standards without changing the main features or damage to the tested object. NDT techniques supply affordable ways of assessing a specimen individually or may be applied to the whole material for testing in a manufacturing system for quality control purposes [2].

There are some advantages and disadvantages inherent to all NDT methods that made them more or less suitable for a particular application in relation to the coverage area, the penetration depth and the problems associated with the interpretation of the results [3]. For instance, ultrasound testing is a mature technique allowing the identification and characterization of deep defects, but requires contact with the object being inspected and the use of water-based couplants (liquid or gel). Radiography testing provides high-resolution images and deep penetration, but requires access to both sides of the object and employs ionizing radiation, which constitutes a risk of radiation exposure. Infrared thermography 
testing allows the inspection of large areas in a fast and contactless manner, but is limited to relatively shallow defects and requires advanced signal/image processing for defect characterization. Eddy current testing (ECT) is effective for certain types of defects (cracks, corrosion, etc.) under several layers of materials, but is limited to conductive materials and is not well suited for the detection of delaminations in composites. It has recently been shown that an alternative electromagnetic method based on a capacitive sensor can be used as an NDT method [2].

For nonconducting materials, NDT methods are not as well developed as compared to those for metallic materials. One approach to evaluate nonconducting materials is to characterize their dielectric properties. The dielectric strength and the dielectric constant are the dielectric properties of nonconducting or low conductivity materials. The voltage that a material can withstand before an electrical breakdown happens is called "dielectric strength" and a measure of the material's capability of storing electric energy is called "dielectric constant" or "permittivity".There exist alternative approaches that are based on the characterization of materials permittivity such as microwave techniques and resonant testing. However, these techniques require expensive equipment and/or complex operating procedures. Capacitive sensing on the contrary is a straightforward and inexpensive approach [2].

The coplanar capacitive technique was first introduced in 2006 [4]. This novel approach, usually referred to as Capacitive Imaging (CI), is an electromagnetic NDT method that uses arrays of electrodes to generate an electric field distribution within the specimen. The electric field distribution can penetrate within dielectric materials and changes in response to the structure of the material under test leading to a variation in the output voltage $[5,6]$. The capacitive technique has shown great potential to inspect a wide range of materials and structures from insulators to conductors [5].

CI technique offers a possible way to overcome some of the restrictions imposed by existing NDT methods. For instance, the technique works in a volume averaging manner and therefore the scattering issue with the ultrasonic method is absent [7]. The affordable cost, straightforward response, noninvasive, no ionizing radiation involved and flexibility in the design of the electrodes provide CI with a great potential to be applied in a extensive range of applications. Furthermore, the coplanar structure allows one-sided inspection [8], which is especially useful when access to both sides of the specimen is limited [9]. Moreover, this is a noncontact method [5] and the liftoff (the air gap between the surface of the electrodes and specimen) can be optimized, leading to an applicable method in the detection of corrosion under insulation (CUI), including the detection of large surface features in metals and therefore the presence of even small amounts of rust [10]. This technique is especially useful in defence where composites are extensively used for both equipment and strengthening structures [11]. These features of coplanar capacitive sensor make it an attractive option for applications in NDT [6], material characterization [8], and imaging [12].

The principle of this technique involves placing two (or more) electrodes over the surface of the sample and then applying an AC voltage between them. This system acts as a capacitor whose altering in capacitance indicates variations in the internal structure (such as the presence of a defect) [13]. Normally, in a regular capacitor, the plates are parallel. When a voltage is applied between these plates, they produce a uniform electric field distribution. This electric field does not restrict itself to the area between the electrodes when the electrodes are gradually open, but extends to a wider space and forms a fringing field [9]. This field expands into the specimen for NDT. Scanning a set of electrodes over the surface of the sample and measuring variations in stored charges for a given voltage creates a map of the changes in the electrical properties within the specimen [7]. The fringing field between the driving and sensing electrodes becomes predominant when the electrodes open to a coplanar plane. This kind of sensor is named a coplanar sensor in literature [9]. Figure 1 shows a schematic diagram showing how the electric field distribution changes when the two capacitor electrodes change from a parallel-plate to a coplanar so that the final 
part produces a fringing field [7]. The coplanar electrodes can be scanned over the surface of a large mechanical structure to investigate the physical properties of the specimen and obtain information.

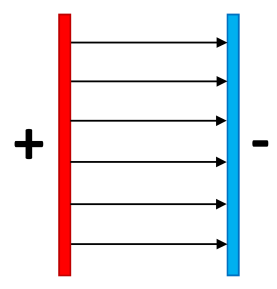

(a)

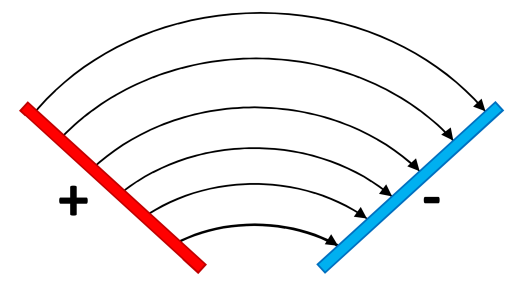

(b)

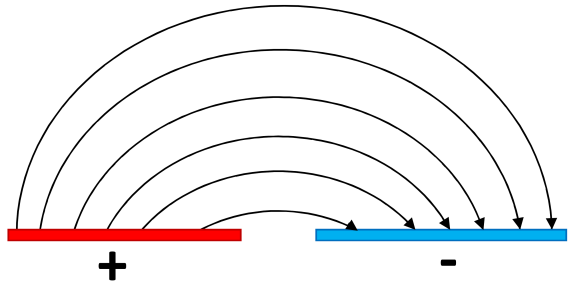

(c)

Figure 1. Schematic of the electric field distribution as electrodes turns from a parallel geometry of the capacitor (left) to coplanar (right): (a) Parallel-plate capacitor whose, (b) Electrodes open up to provide, and (c) One-sided access to the specimen.

Such as some other NDT methods, the capacitive technique is sensitive to liftoff, which refers to the air gap between the surface of the coplanar electrodes and specimen which causes a decrease in the capacitance between the electrodes and hence a reduction in output voltage. In addition, it reduces the extent of penetration of the electric field into the sample which leads to a decrease in the depth of penetration [9]. The lift-ff should therefore be as small as possible to achieve greater signal strength and sensing depth (especially for investigating dielectric material) [14] with no need to use couplant materials.

The sensor manufacturing steps include material selection for electrodes, the insulation layer of the surface of the electrodes, the sensor substrate, and eventually the selection of a production technique. The electrodes are made of materials with high electrical conductance, such as copper. A thin insulation layer (with a few micrometres in thickness) is usually placed on the surface of the electrode to prevent direct contact of the electrodes with the specimen and to protect the electrode surface against scratches. The thickness of the insulation layer and the sensor substrate influence the electric field strength and depth of penetration, therefore, need to be optimized. Several manufacturing techniques of the capacitive sensor, including microelectromechanical systems (MEMS) [15], printed circuit boards (PCB) and manual production can be selected depending on sensor dimensions and costs [9].

To evaluate the sensor performance, it is required to explain the general design parameters. Therefore, in this paper, firstly the design factors such as the geometry of the electrodes, the number of electrodes and their arrangement, shielding plate and guard electrode of the capacitive sensor are presented. The electric field distribution from a coplanar capacitive sensor in the different condition of a specimen, which are the sound sample, the sample with a crack on the surface, and the sample with subsurface defect, was considered by 3D finite element (FE) modeling in COMSOL Multiphysics software. The real sample, a Plexiglas plate with defects at different depths, was simulated using FE modeling and the electric field strength was measured. It was shown that the different depths of the defect have different electric field strength and distribution. These results approved by physical experiments and It was illustrated that the electric field strength and distribution changed with respect to the defect depth in agreement with experimental data.

\section{Sensor Design}

There are several parameters in sensor design for assessing the performance of a sensor. One design parameter may impact several aspects of sensor performance. On the other hand, more than one design factor should be considered in order to achieve the desired sensor performance. Therefore, knowing how design parameters affect sensor performance leads to sensor optimization for a specific application. Additionally, instrumentationrelated issues should be considered in order to achieve the right measurements of a sensor [9]. The most important design parameters for a coplanar capacitive sensor include 
the electrode geometry, the number of electrodes and their arrangement, shielding plate and guard electrode.

\subsection{Electrodes Geometry}

The performance of a coplanar capacitive sensor is primarily determined by its geometry, which includes the size, shape, and separation distance between the electrodes [16]. They are the most important parameters which affect the electric field strength and the penetration depth of the probe [17]. A bigger electrode size provides a deeper penetration depth; however, it reduces the image resolution in imaging applications since it samples a bigger area of the specimen. The shape of electrodes can be a simple form such as square, rectangular, triangular shape, or complex shape, like a comb or spiral shape. Free space width between adjacent electrodes refers to the separation distance between the electrodes and has a significant impact on the measured output. Therefore, the geometry of the electrode influences the sensor performance in signal strength, depth of penetration and sensitivity of the measurement and need to be optimized and selected with care $[9,18]$.

\subsection{Number of Electrodes and Their Arrangement}

The number of electrodes proper to a given application depends on the geometry of the sample and its physical parameter. For some investigations, like proximity or displacement measurement application, in which there is a direct relationship between capacitance measurements and the distance of a specimen, a single sensing element, like an annular sensor is often enough to infer the proximity of the specimen from capacitance measurement. For more complex situations, such as imaging or NDT applications, an array of electrodes would be more adequate. In addition, the arrangement of the electrodes and the available space in the system to place the electrodes must be considered $[9,16]$.

\subsection{Shielding and Guarding}

Generally, a shielding plate and a guard electrode are employed to shape the electric field, and more importantly to eliminate stray capacitance and noise [17]. Indeed, a shielding plate, which is placed on top of the main electrodes, is needed to attenuate the undesirable electric field, to eliminate parasitic capacitance and noise in the sensing electrodes; and a guard electrode, which surrounds the main electrodes and between them, will be used to prevent the electric field lines to go directly to the sensing electrode. In this way, they have to travel further into the specimen to reach the sensing electrode, thus, the depth of penetration will increase as shown in Figure 2. Usually, the shielding plate and guard electrode are held at ground potential and different types of them can be used depending on the capacitor measurement circuit [18].

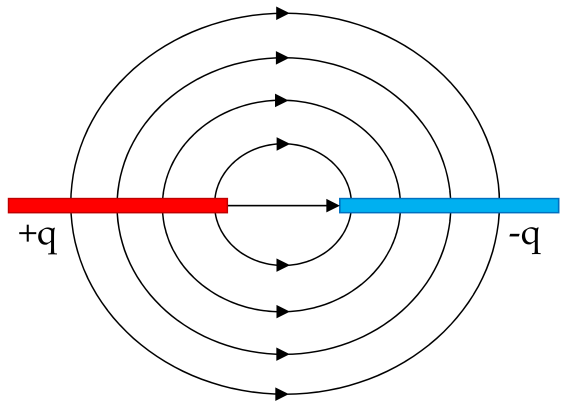

(a)

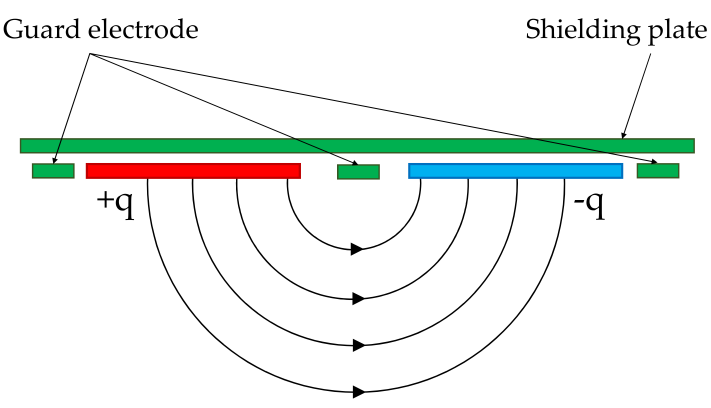

(b)

Figure 2. Schematic diagrams for capacitive probe with and without shielding plate and guard electrodes: (a) Cross-section of the capacitive probe without shielding plate and guard electrodes and (b) Cross-section of the capacitive probe with shielding plate and guard electrodes. 


\section{The Penetration Depth of the Capacitive Technique}

For a coplanar capacitance sensor, the penetration depth is often defined as the maximum distance in the vertical direction to the electrodes that produce a detectable variation in the sensor output. It is an important parameter to assess the sensor performance because it indicates how far the sensor can sense [19]. The penetration depth is determined by the material properties (such as permittivity), the noise level of the equipment, and the electrode geometry (shape, size, and arrangement) $[9,18]$. The material permittivity has certainly an important impact on the penetration depth, the higher the permittivity the faster the decay of the electric field. However, the primary factor having an influence on the penetration depth of a given material is the geometry of the driving and sensing electrodes. In the case of coplanar capacitive sensors, penetration depth determination is difficult due to the different types of electrode geometry and the complexity of the fringing electric field. A practical and straightforward manner to determine the effective penetration depth of the capacitive sensor is to place the probe over the inspected specimen and gradually move it away from its surface while measuring the capacitance between driving and sensing electrodes [9]. This technique is different from the other NDT method such as eddy currents, where the variation of frequency leads to a change in the depth of penetration while the sensing depth of capacitive sensors mainly depends on the probe geometry and properties of the specimen [18].

\section{Numerical Simulation and Experimental Work}

The capacitive probe is the key part of the instrumentation and requires to be designed carefully. For sensors with a constant overall electrode area, the penetration depth increases with the distance between the electrodes. A larger distance between electrodes will however decrease the signal strength (the measured output) as the coupling between the driving and sensing electrodes would be weaker and would reduce the image resolution as they are sampling a larger area of the sample and more importantly it is physically disallowed if the overall probe size is constant. To balance these trade-offs, triangular-shaped electrodes can be applied [20]. This electrode geometry is an interesting choice for different defect detection in various material as it would likely allow deep penetration into the specimen, with a symmetrical electric field distribution. As stated in the previous section, the electric field distribution is a function of the electrode geometry and so this aspect has to be designed with care [5]. The coplanar electrodes with the same overall sensing areas are used in this work, as shown in Figure 3, namely capacitive probes with back-to-back triangular electrodes. Generally, this pair of triangular electrodes could be specified by the overall size, the base $(b)$, the height $(\mathrm{h})$ of each triangle and the separation distance between the closest points of the two triangles (s). In this work, the triangular electrodes have the same overall size with $\mathrm{b}=15.0 \mathrm{~mm}, \mathrm{~h}=15.0 \mathrm{~mm}$ and $\mathrm{s}=4.0 \mathrm{~mm}$. In addition, a shielding plate and guard electrode are employed for this coplanar capacitive sensor. Note that the field distribution predicted by the finite element (FE) model, as discussed below, is determined primarily by these geometric factors in the model. 


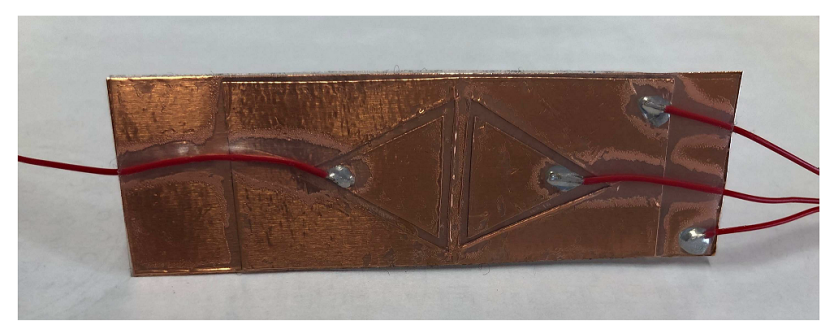

(a)

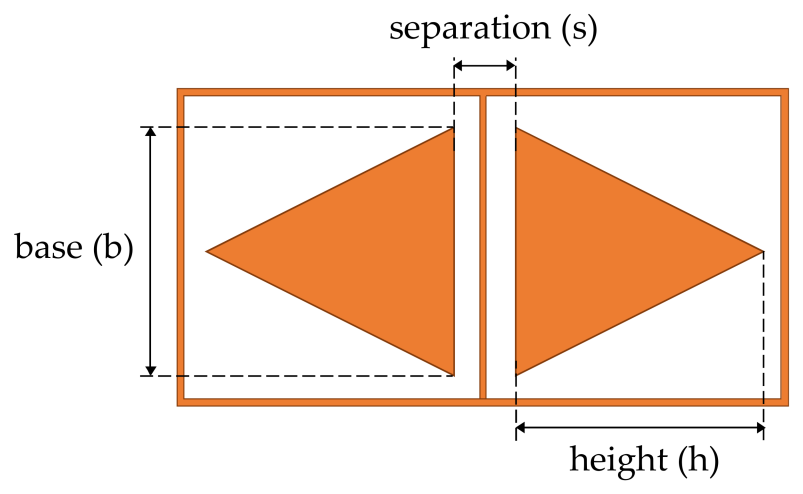

(b)

Figure 3. (a) Photograph of the pair of triangular electrodes and (b) Diagram of the capacitive sensor as viewed from the top surface.

\subsection{Numerical Simulation}

Conceptually, the capacitive method is straightforward. The electrodes provide an electric field distribution when an AC voltage is applied between the driving and sensing electrodes. The presence of the specimen and any variation in its properties (such as the presence of a defect) within the area covered by the electric field will change the field pattern distribution. A distortion in the electric field causes a change in the charge induced in the sensing electrode, and this change in the charge can be used for defect detection purposes [7]. The resulting signal is sent to standard instruments such as charge amplifiers that increase the signal-to-noise ratio (SNR) and allow small changes in the signal to be recorded, processed and outputted as a DC voltage. Since the presence of delaminations, cracks and other imperfections changes the electric field distribution in various distinctive ways, a basis for detecting defects can be established [5].

In order to understand the way electric field distributions are produced by the electrodes, and to identify how they may interact with the materials of interest, it was felt important to model the expected behavior. In this paper, we want to demonstrate the distribution of the electric field and how changes in the specimen might affect the output. Finite element (FE) modeling enables valuable information (such as visualization of the results) to towards analyzing the performance of the capacitive sensor more easily. Although finite element methods are well established in engineering calculation, care must be taken to secure suitable mesh densities in critical regions, in particular near to the source of field singularities [21]. The major source of errors is the discretization processes of the conductor segments bordering different dielectrics and of the dielectric interfaces. However, there is no need to do the complicated mathematical process in order to simulate the defects in the samples in the finite element method. This is a useful tool for predicting the fields from capacitive electrodes and how these fields are likely to interact with different materials and defects $[5,22]$. Therefore, finite element (FE) modeling has been employed to predict the distribution of the electric field from capacitive electrodes and signal changes that are likely to be generated under experimental conditions.

Theoretical simulations were conducted using COMSOL ${ }^{\mathrm{TM}}$ Multiphysics FE package, and using its AC/DC module, electric currents (ec)-type, which can be used to model the distribution of the electric field in different situations. The purpose of this analysis is to observe the field distribution and the variation of electric field strength as a function of the sample properties such as existing defects and depths of a defect. The electric field distribution for all situations has been analyzed using a three-dimensional (3D) finite element method.

In this model, the driving and sensing electrode were driven by a $+5.0 \mathrm{~V}$ and $-5.0 \mathrm{~V}$, respectively, with a $100 \mathrm{kHz}$ sinusoidal signal. The shielding plate and guard electrode were set to ground potential. Based on this, there is an electric field between the positive and negative electrodes and the electric field distribution is formed in the local region in 
the specimen under the electrode pair and the liftoff between the sample and probe. A pair of a coplanar triangular electrode is placed above the surface of a specimen with $1.0 \mathrm{~mm}$ liftoff, and the setup is enclosed in a $120 \mathrm{~mm} \times 120 \mathrm{~mm} \times 120 \mathrm{~mm}$ block centred at the point $(x=0, y=0, z=0)$. This block was a zero-charge boundary airbox and defined to be the computational domain, as shown in Figure 4a. A physics-controlled mesh was used and the mesh generation density is set to "Finer". Providing the environment and necessary system parameters the mesh is generated in the model as shown in Figure $4 \mathrm{~b}$. Figure $4 \mathrm{c}$ shows the solved back-to-back triangular capacitive sensor model for a sound specimen. The contour lines on top of the specimen represent electric potential values.

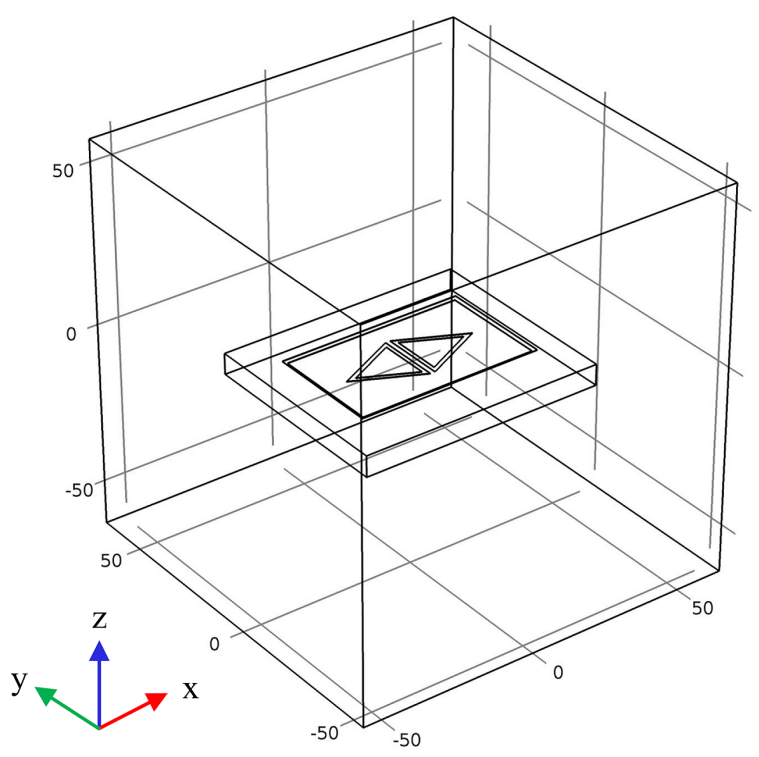

(a)

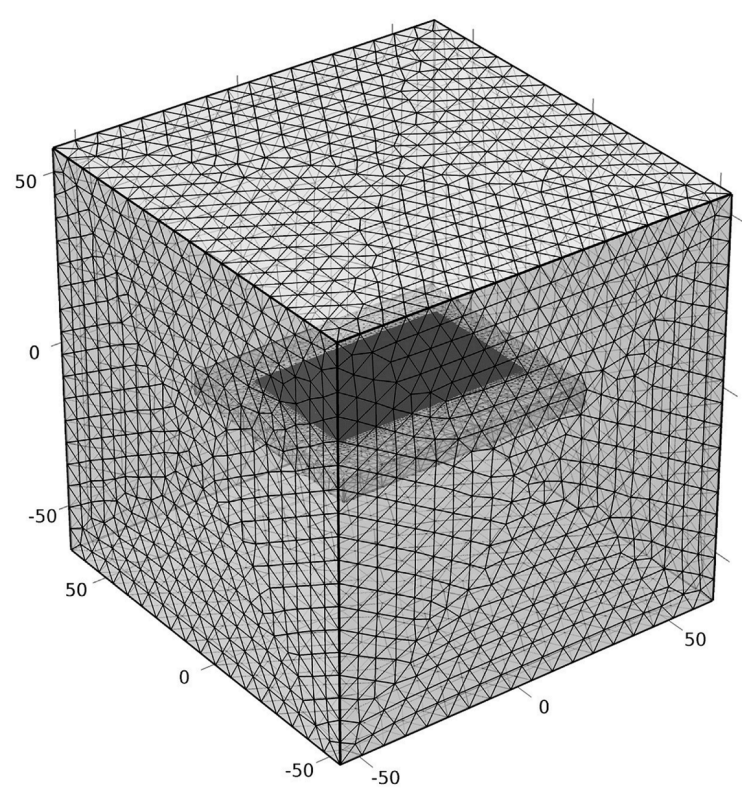

(b)

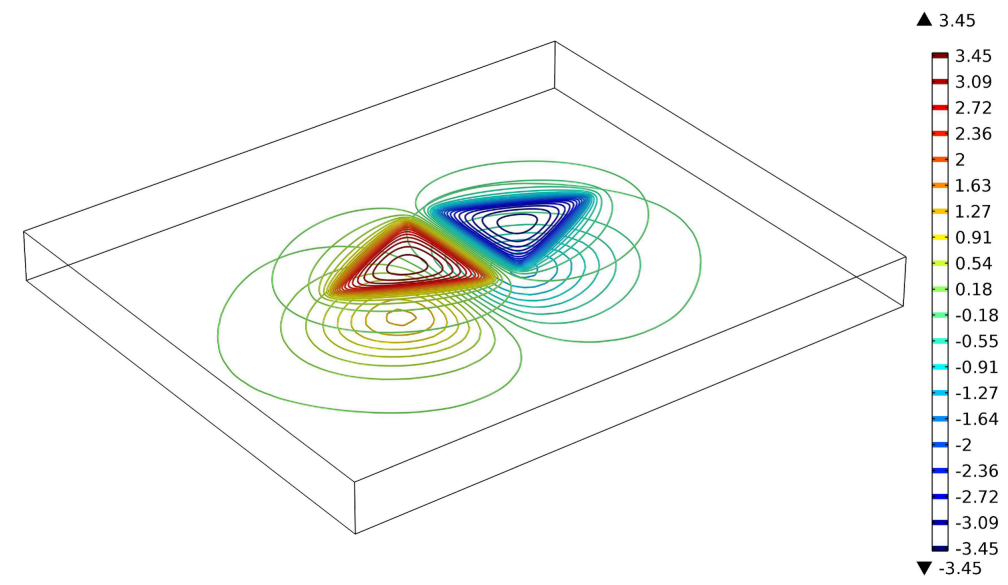

(c)

Figure 4. 3D model of the finite element modeling: (a) the computational domain $(120 \mathrm{~mm} \times 120 \mathrm{~mm} \times 120 \mathrm{~mm})$ with a coplanar capacitive probe, (b) model after finite element mesh generation, and (c) The electric potential contour lines on the surface of the sound specimen.

In the coordinate system illustrated in Figure 5, based on which the results will be shown henceforth, the surface of the electrodes is centred at $(y=0$ or $z=0)$. $Y=0$ plane (or $x z$ plane) is the cross-sectional plane along the long axis of symmetry of the capacitive sensor, as shown in Figure $5 \mathrm{a}$, and $z=0$ plane (or $x y$ plane) is the parallel plane under the probe surface at the sample surface, as shown in Figure $5 \mathrm{~b}$. To clarify the results, the plane coordinate systems for the two types of cross-sections are presented in Figure $5 c$. 


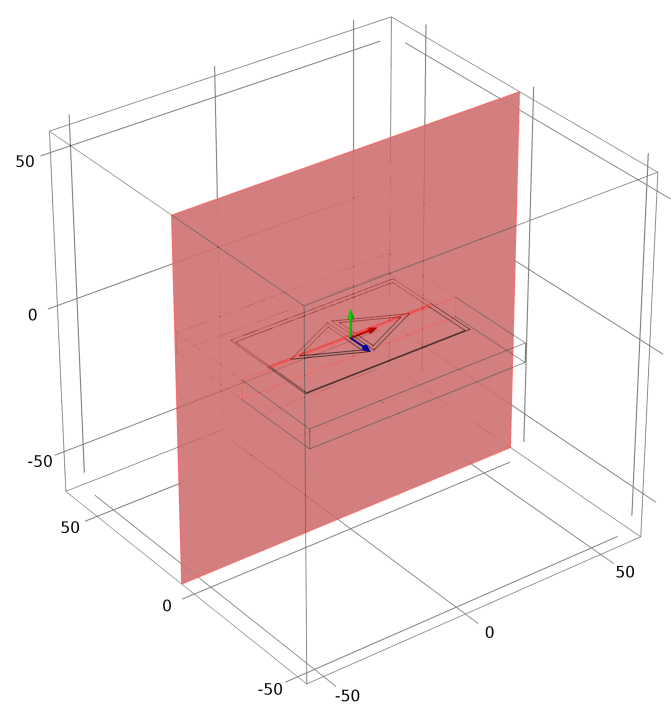

(a)

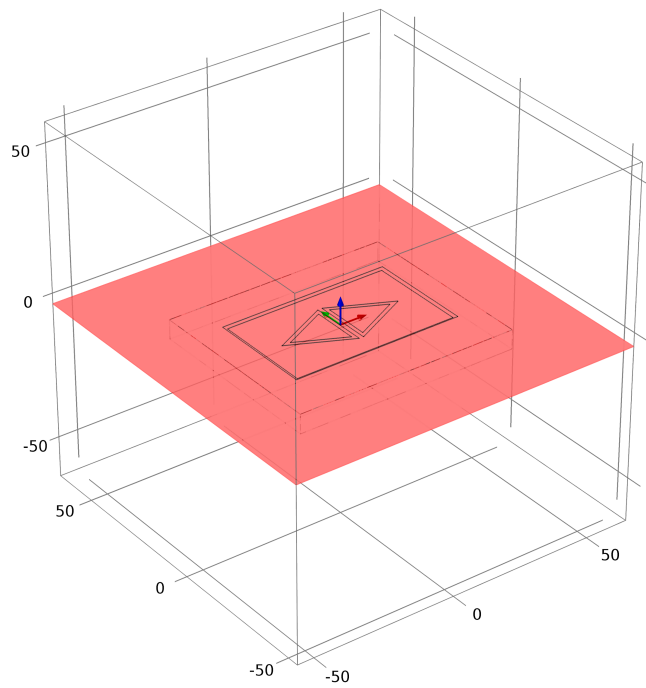

(b)

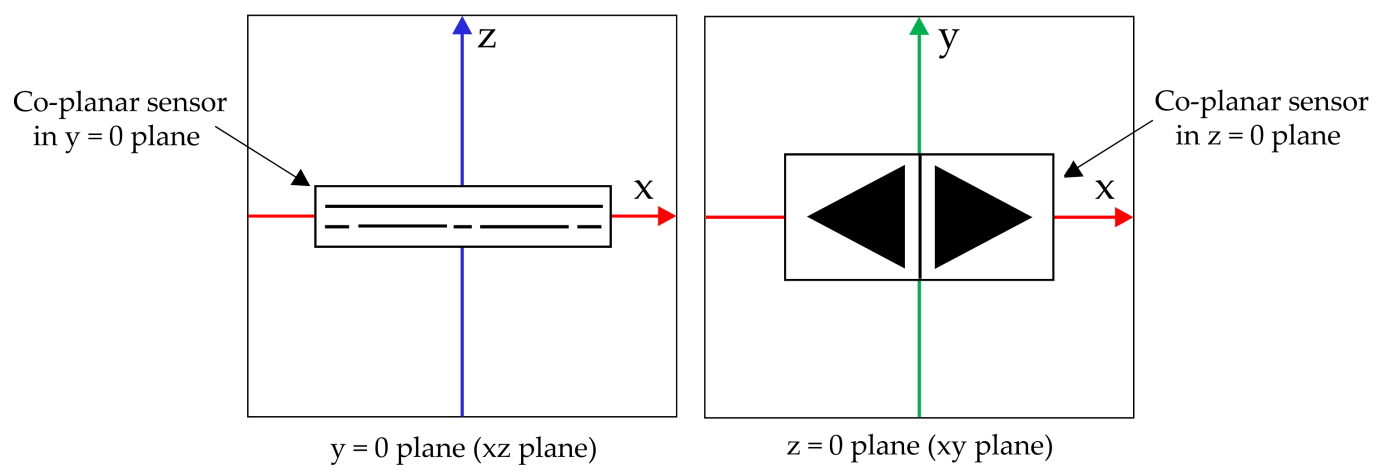

(c)

Figure 5. The cross-sectional plane along the long axis of symmetry of the capacitive probe: (a) $y=0$ plane ( $x z$ plane), (b) $z=0$ plane ( $x y$ plane), and (c) plane coordinate systems for the two kinds of cross sections.

Figure 6 shows a sample of the electric field distribution prediction of the 3D finite element models for the coplanar capacitive electrodes and how these fields behave under different conditions. This figure is a contour plot of the electric field in the $y=0$ plane. Figure 6a shows this example for a sound zone of a specimen, Figure $6 \mathrm{~b}$ shows a sample of output for a defective zone filled with air, and Figure $6 \mathrm{c}$ shows the electric field distribution for a specimen with a narrow crack on the surface. It can be seen that the presence of defects distorts the electric field within and around the regions of discontinuity in the sample and this leads to a variation in the detectable signal on the sensing electrode. This is because of the different dielectric properties such as permittivity (or dielectric constant) presumed for the sample and defects. Therefore, the model shows how the capacitive sensor detects the defects due to the different dielectric properties between the sample and defect. 


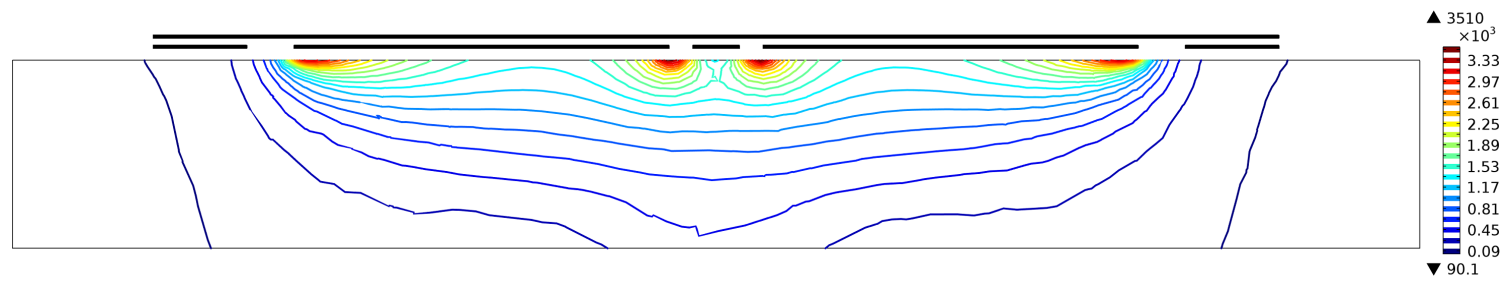

(a)

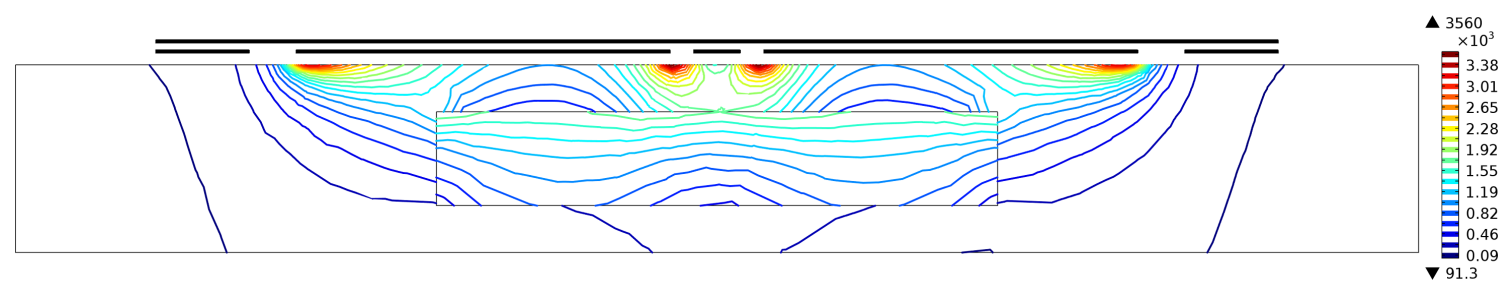

(b)

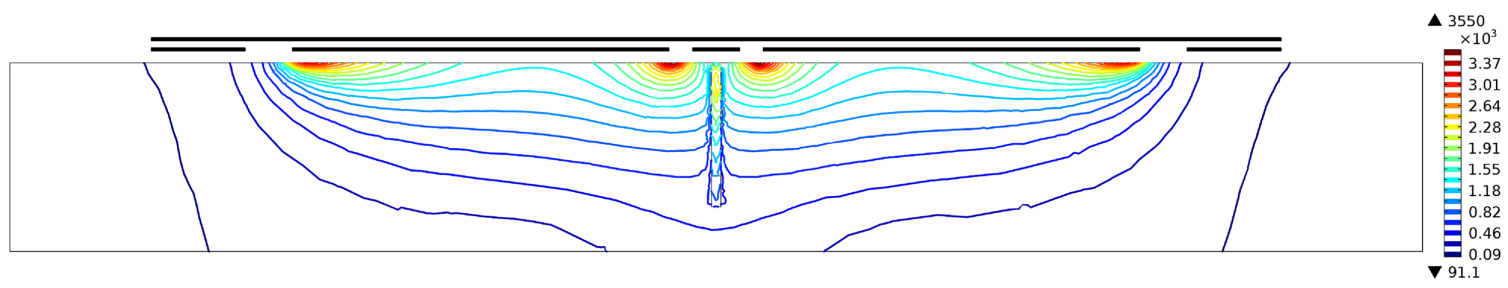

(c)

Figure 6. Simulations of the electric field distribution $(\mathrm{V} / \mathrm{m})$ for a sample in $y=0$ plane: (a) no defect, (b) void under the surface, and (c) a sample with a narrow crack on the surface.

Simulations were conducted to determine the effect of various depths of subsurface defects on the electric field strength. The real nonconducting sample, a Plexiglas plate containing three subsurface air-filled cylindrical voids at different depths, was simulated. Figure 7 shows a surface plot of the electric field of the back-to-back triangular electrodes for the different defect depth in the $z=0$ planes, including Figure $7 \mathrm{a}$ a sound sample, (b) a defect in $0.5 \mathrm{~mm}$ depth, (c) a defect in $1.0 \mathrm{~mm}$ depth, and (d) a defect in $1.5 \mathrm{~mm}$ depth. By comparing the results, it can be inferred the presence of the air-filled defect at different depths causes a different distortion of the electric field and this would lead to a detectable change in the output. This is caused by the different values of permittivity (or dielectric constant) assumed for the solid sample (Plexiglas, permittivity $=3.4$ ) and air (permittivity $=1.0$ ). Thus, the model shows how the capacitive probe detects subsurface defects due to a permittivity difference between the sample and defect.

It is obvious from these results that the electric field strength decreases as the depth of the defect increases. In other words, the penetration depth and strength of the electric field vary for different depths of the defect. This is confirmed by the experimental result to be shown later in this work. Moreover, these figures show that different depths of a defect create a different electric field pattern which can be used to estimate the defect depth. 


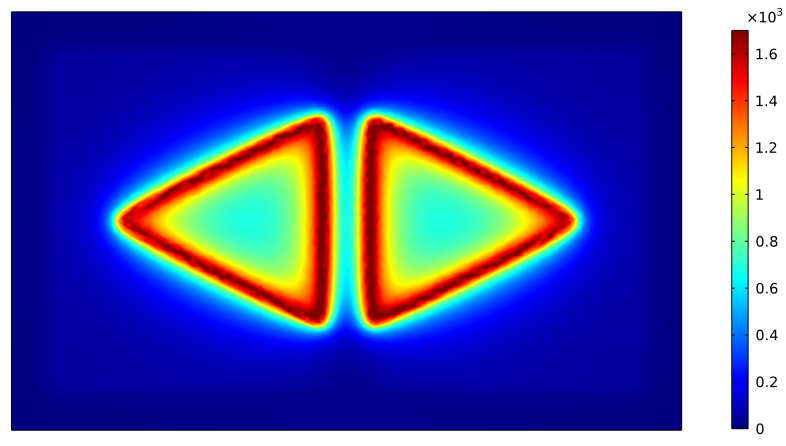

(a)

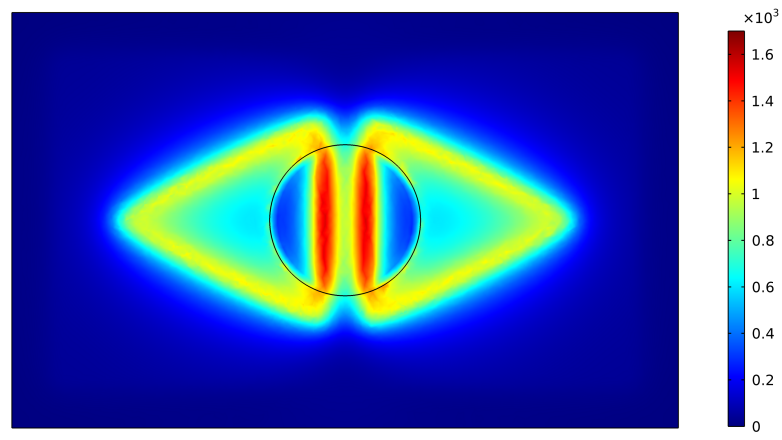

(b)

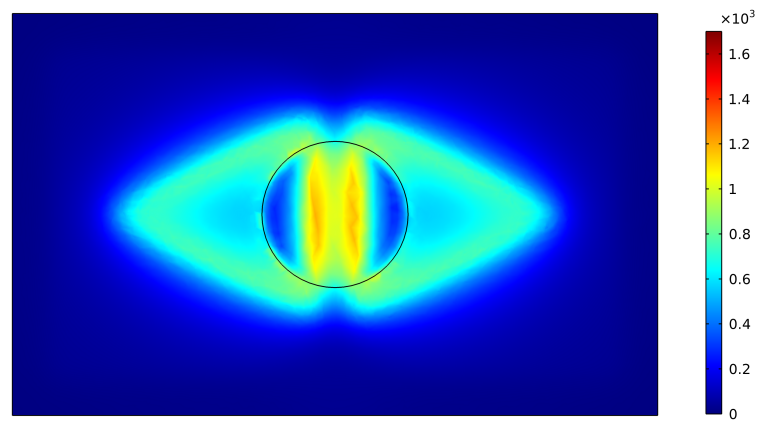

(c)

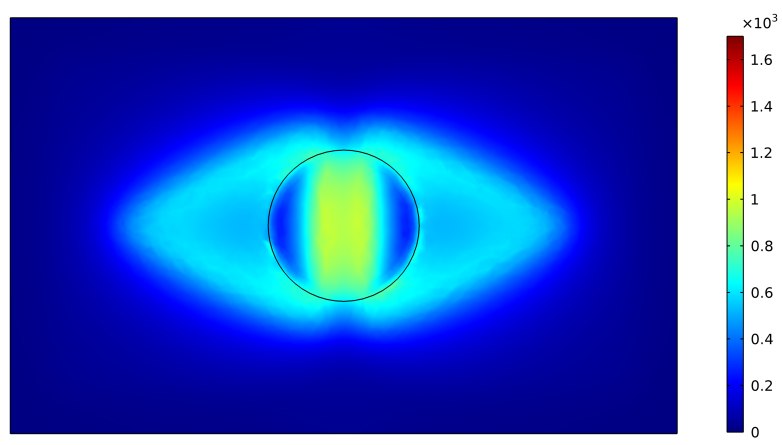

(d)

Figure 7. Simulations of the electric field distribution $(\mathrm{V} / \mathrm{m})$ for a sample in $z=0$ plane: (a) no defect, (b) a defect at $0.5 \mathrm{~mm}$ depth, (c) a defect at $1.0 \mathrm{~mm}$ depth, and (d) a defect at $1.5 \mathrm{~mm}$ depth. 


\subsection{Experimental Results}

Figure 8 illustrates the experimental arrangement used for the capacitive technique experiments presented in this work. The experiments use a capacitance setup that is composed of a single pair of back-to-back triangular electrodes made of copper plates as shown in Figure 3. A shielding plate is placed on its top side to ensure that the radiated electric field emanates predominantly in the direction towards the specimen and to provide protection against the effect of the ambient stray fields on the output results. In addition, a guard electrode is applied to increase the penetration depth of the sensor. One of the electrodes is used as a driver electrode and the other one as a receiver. The driving electrode was excited by a sinusoidal voltage of $10 \mathrm{~V}$ amplitude peak-to-peak at a frequency of $100 \mathrm{kHz}$ and the shielding plate and guard electrode are connected to the ground potential. To generate an electric field, one of the plates is employed as a transmitter and connected to Ectane. Ectane is a multitechnology, powerful and compact instrument for generating and analyzing signals. The frequency range of this instrument is $5 \mathrm{~Hz}$ up to $10 \mathrm{MHz}$ and the generator output is up to $20 \mathrm{~V}$, peak to peak. A differential amplifier is placed inside Ectane to process the received signal successively. The output signal from the Ectane is a simple DC voltage level that is proportional to the instantaneous value of the dielectric property of the material averaged over the field distribution within the material.

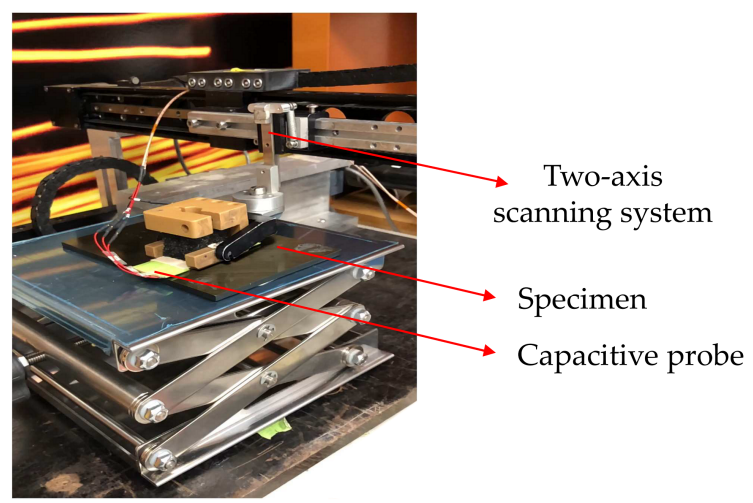

(a)

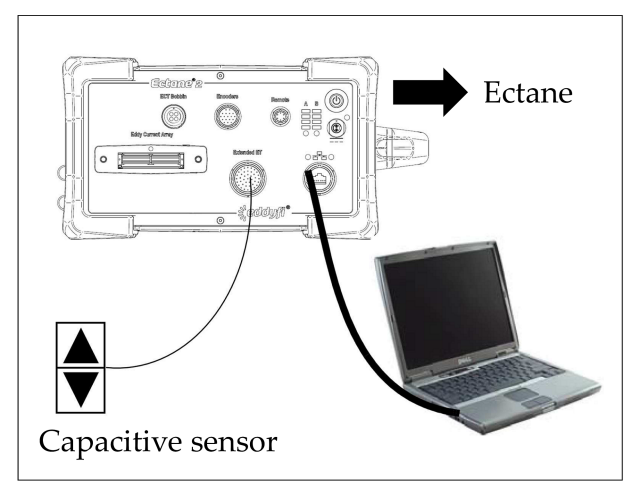

(b)

Figure 8. (a) The experimental arrangement used for the capacitive imaging and (b) typical configuration: Ectane with the capacitive sensor.

The hidden depth of the defect is also a factor affecting the coplanar capacitive sensor performance. To investigate the effects of it in nonconducting specimens, a set of experiments were carried out on a $6.5 \mathrm{~mm}$ thick Plexiglas plate. The plate contains three subsurface defects of $12 \mathrm{~mm}$ diameter located at different depths $(0.5 \mathrm{~mm}, 1.0 \mathrm{~mm}$, and $1.5 \mathrm{~mm}$ ). Figure $9 \mathrm{a}$ illustrates the schematic diagram of the sample. The coplanar CI probe was scanned over the Face (A) (the face without the defects) using a two-axis scanning system $(x-y$ table). In this scan, the coplanar capacitive sensor was held $1.0 \mathrm{~mm}$ off the surface of the specimen (a constant liftoff $=1.0 \mathrm{~mm}$ ) and scanned over a $40 \mathrm{~mm} \times 160 \mathrm{~mm}$ area with a $0.2 \mathrm{~mm}$ step in the $x$-axis and $1.0 \mathrm{~mm}$ in the $y$-axis. The speed of the probe was set to $10 \mathrm{~mm} / \mathrm{s}$.

Figure $9 \mathrm{~b}$ is the image obtained by scanning the coplanar capacitive sensor over the surface of the Plexiglas sample. The technique easily detected these defects, and this is due to the different dielectric properties between the material of the specimen (Plexiglas) and the defect (air). It can be seen from the result, the defect at $0.5 \mathrm{~mm}$ depth, which is the shallowest defect in the Plexiglas specimen, appeared as a darker colour, especially a darker red colour which is representing the highest output voltage in this result. Likewise, the other two defects appeared as a lighter area, depending on their depth representing lower output voltage values. This result is in good qualitative agreement with the result of the simulations as seen in Figure 7. 


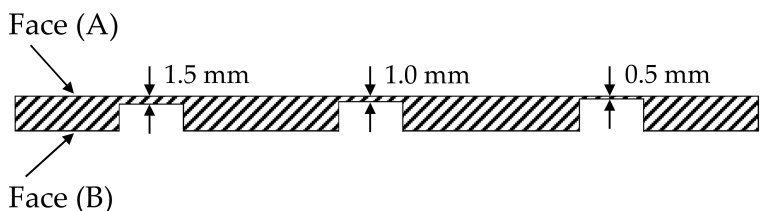

(a)

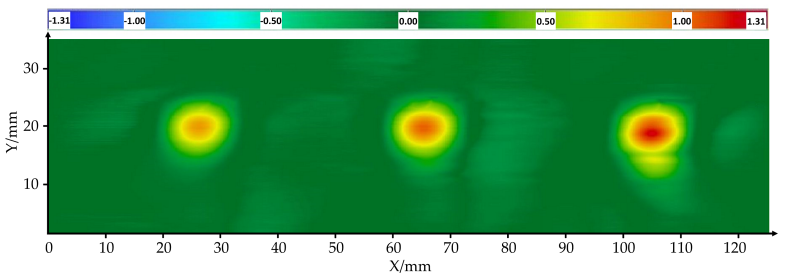

(b)

Figure 9. (a) Diagram of the Plexiglas specimen with three holes of different depths and (b) the capacitive image based on voltage values. In this image obtained from the scanning of the probe on the specimen, the striking feature is the red and yellow area, which suggests the successful detection of the defects. It can be seen that the colour of the defect-free area is green. (The reflection on the third rightmost defect is because of the mechanical adjustment.)

It can be inferred that when a specimen is placed under the coplanar capacitive electrodes, most of the electric field from the sensor penetrates into the specimen and then ends on the sensing electrode. The dielectric properties of the specimen and defects influence the electric field distribution and, hence, on the electric potential of the sensing electrode. Therefore, an existing defect in the specimen will change the electric field pattern, which leads to the changing in the detectable signal of the sensing electrode. It is worth mentioning the depth of penetration is determined mainly by the geometry of the sensor, the electric properties of the specimen, and the liftoff.

\section{Conclusions}

A feasibility study of the coplanar capacitive sensing technique which has certain advantages for NDT applications was explored through a numerical simulation and experimental tests. Numerical finite element (FE) analysis was conducted to demonstrate the validity of the proposed defect detection technique. The 3D FE modeling has demonstrated that the electric field distribution of a selected electrode pair penetrate into dielectric materials and can be used to detect the presence of defects in the form of air voids and crack. The preliminary investigation into the appliance of capacitive sensing for the detection of hidden holes within the specimen has been successfully shown. Experimental results on the Plexiglas plate containing subsurface holes of various depths gave a sign of the response of the technique. A good qualitative agreement was observed between the experimental and simulations result. The results obtained by the coplanar capacitive method illustrate that the technique is noncontact, noninvasive, and only requires single-sided access to the specimen. The couplant such as gel or water is not required, and there is no need for specific surface preparation. In addition, there are no radiation exposure problems. Therefore, this technique has the potential to be used in many NDT application areas where traditional methods may have difficulty.

In practice, the condition is typically more complicated compared to the sample inspected during this work. Defect specifications will be unknown, and voids could also be poorly defined. Further development of the capacitive sensor is required to meet various practical needs and provide enhanced diagnostic information, e.g. systematic identification and characterization of defects. It should be emphasized that the depth of the penetration is mainly determined by the probe geometry, the electric properties of the specimen and the liftoff distance between the surface of the probe and the specimen, this will be further studied in future work.

Author Contributions: Conceptualization, methodology, formal analysis, software, experimental setup and data acquisition, validation, investigation, and writing—original draft preparation, F.A.-M.; methodology, formal analysis and experimental setup, S.S.; writing-review and editing, T.F., C.I.-C., and X.P.V.M.; supervision, X.P.V.M.; All authors have read and agreed to the published version of the manuscript.

Funding: This research is funded by the Natural Sciences and Engineering Research Council of Canada (NSERC)—Discovery Grant Program, the Canadian Foundation for Innovation (CFI) and 
Canada Research Chair Program. The support of the NSERC CREATE-oN DuTy! Program is also acknowledged.

Institutional Review Board Statement: No institutional board review was necessary for this project. Informed Consent Statement: Not applicable.

Data Availability Statement: Data sharing not applicable.

Acknowledgments: The authors would like to thank and express their appreciation to the Eddyfi technologies and Vision Laboratory team at Laval University for supporting this research. They also would like to thank Hugo Lemieux and Eric Morissette from Eddyfi Technologies for their scientific advice.

Conflicts of Interest: The funders had no role in the design of the study; in the collection, analyses, or interpretation of data; in the writing of the manuscript, or in the decision to publish the results.

\section{References}

1. Gholizadeh, S. A review of non-destructive testing methods of composite materials. Procedia Struct. Integr. 2016, 1, 50-57. [CrossRef]

2. Chen, T. Capacitive sensors for measuring complex permittivity of planar and cylindrical structures. Lowa 2012. [CrossRef]

3. Nassr, A.A.; Ahmed, W.H.; El-Dakhakhni, W.W. Coplanar capacitance sensors for detecting water intrusion in composite structures. Meas. Sci. Technol. 2008, 19, 075702. [CrossRef]

4. Liu, Z.; Liu, H. Experimenting capacitive sensing technique for structural integrity assessment. In Prodeedings of the 2017 IEEE International Conference on Industrial Technology (ICIT), Toronto, ON, Canada, 22-25 March 2017; pp. $922-927$.

5. Yin, X.; Diamond, G.; Hutchins, D. Further investigations into capacitive imaging for NDE. Insight-Non-Destr. Test. Cond. Monit. 2009, 51, 484-490. [CrossRef]

6. Diamond, G.G.; Hutchins, D.A. A new capacitive imaging technique for NDT. In Proceedings of the European Conference on NDT, Berlin, Germany, 25-29 September 2006; pp. 1-8.

7. Yin, X.; Hutchins, D. Non-destructive evaluation of composite materials using a capacitive imaging technique. Compos. Part B Eng. 2012, 43, 1282-1292. [CrossRef]

8. Mamishev, A.V.; Sundara-Rajan, K.; Yang, F.; Du, Y.; Zahn, M. Interdigital sensors and transducers. Proc. IEEE 2004, 92, 808-845. [CrossRef]

9. Hu, X.; Yang, W. Planar capacitive sensors-designs and applications. Sens. Rev. 2010. [CrossRef]

10. Yin, X.; Hutchins, D.A.; Chen, G.; Li, W. Detecting surface features on conducting specimens through an insulation layer using a capacitive imaging technique. NDT E Int. 2012, 52, 157-166. [CrossRef]

11. Sharma, D.; Goyal, S.; Khanna, R.; Mukherjee, A. Non-destructive testing of materials using capacitive sensing technique. Mit Int. J. Electron. Commun. Eng. 2011, 1, 73-77.

12. Cheng, B. Security Imaging Devices with Planar Capacitance Sensor Arrays. Ph.D. Thesis, University of Manchester, Manchester, UK, 2008.

13. Dérobert, X.; Iaquinta, J.; Klysz, G.; Balayssac, J.P. Use of capacitive and GPR techniques for the non-destructive evaluation of cover concrete. NDT E Int. 2008, 41, 44-52. [CrossRef]

14. Yin, X.; Li, C.; Li, Z.; Li, W.; Chen, G. Lift-off effect for capacitive imaging sensors. Sensors 2018, 18, 4286. [CrossRef] [PubMed]

15. Chen, Z.; Luo, R.C. Design and implementation of capacitive proximity sensor using microelectromechanical systems technology. IEEE Trans. Ind. Electron. 1998, 45, 886-894. [CrossRef]

16. Yin, X.; Chen, G.; Li, W.; Huthchins, D. Design and characterization of planar capacitive imaging probe based on the measurement sensitivity distribution. In AIP Conference Proceedings; American Institute of Physics: College Park, MD, USA, 2013; Volume 1511, pp. 1586-1592. [CrossRef]

17. Li, X.B.; Larson, S.D.; Zyuzin, A.S.; Mamishev, A.V. Design principles for multichannel fringing electric field sensors. IEEE Sens. J. 2006, 6, 434-440. [CrossRef]

18. Yin, X.; Hutchins, D.A.; Chen, G.; Li, W. Preliminary studies on the design principles of capacitive imaging probes for nondestructive evaluation. Int. J. Appl. Electromagn. Mech. 2013, 42, 447-470. [CrossRef]

19. Chen, D.; Hu, X.; Yang, W. Design of a security screening system with a capacitance sensor matrix operating in single-electrode mode. Meas. Sci. Technol. 2011, 22, 114026. [CrossRef]

20. Yin, X.; Hutchins, D.A.; Chen, G.; Li, W.; Xu, Z. Studies of the factors influencing the imaging performance of the capacitive imaging technique. NDT E Int. 2013, 60, 1-10. [CrossRef]

21. Costamagna, E.; Di Barba, P. Inhomogeneous dielectrics: Conformal mapping and finite-element models. Open Phys. 2017, 15, 839-844. [CrossRef]

22. Meng, G.; Jaworski, A.J.; Kimber, J.C. A multi-electrode capacitance probe for phase detection in oil-water separation processes: Design, modelling and validation. Meas. Sci. Technol. 2006, 17, 881. [CrossRef] 